\title{
Chirped pulse amplification in an erbium-doped fiber oscillator/ erbium-doped fiber amplifier system
}

\author{
Michelle L. Stock and Gerard Mourou \\ Center for Ultrafast Optical Science, University of Michigan, Ann Arbor, MI 48109, USA
}

Received 29 October 1993

\begin{abstract}
Chirped pulse amplification in erbium fibers is demonstrated. A passively mode locked erbium-doped fiber laser source provides the seed pulses for an érbium-doped fiber amplifier. The system produces femtosecond pulses at a repetition rate of $33 \mathrm{MHz}$ with an energy content as high as $1.4 \mathrm{~nJ}$.
\end{abstract}

As in bulk amplifiers, the onset of nonlinear effects when amplifying ultra-short pulses in fiber amplifiers has been pointed out and investigated by several groups, specifically in erbium-doped fiber amplifiers (EDFAs) [1-3]. Researchers have in fact used the Kerr nonlinearity in conjunction with group velocity dispersion in order to create even shorter pulses via higher-order soliton formation [4,5]. However, as Hodel et al. [3] point out, efficient distortionless amplification of soliton pulses in EDFAs is actually prevented by the presence of Raman selfscattering and, beyond the limit of adiabatic amplification, by the break-up of pulses due to frequency dependent gain, self-phase modulation and dispersion. Hence it has been suggested to avoid nonlinearities in the amplification process to fully exploit the energy storage capacity of rare-earth amplifiers.

Here, we demonstrate the application of chirpedpulse amplification (CPA) [6] to the amplification of ultra-short pulses generated by an optical fiber oscillator. This erbium-doped fiber based, compact system should in principle be able to produce femtosecond pulses with energies up to a few microjoules, the saturation energy of EDFAs [2]. This compares quite favorably to diode amplifiers, which are only capable of saturation energies of approximately $100 \mathrm{pJ}$ [7]. The technique of CPA involves temporally stretching a short pulse through a dispersive delay prior to amplification. The pulse peakpower is thereby reduced resulting in a linear am- plification process. The amplified pulse is then recompressed by passing it through a dispersive delay line opposite in effect to the stretcher. In the absence of gain narrowing the original pulse-width can theoretically be obtained.

The experimental set-up is shown in fig. 1. An environmentally stable Kerr-type mode locked erbium fiber laser [8] is the source of seed pulses to the amplifier. This oscillator generates near band-width limited $250 \mathrm{fs}$ pulses at a repetition rate of $33 \mathrm{MHz}$ and a center wavelength of $1.567 \mu \mathrm{m}$ with an energy content of up to $100 \mathrm{pJ}$. The autocorrelation and spectrum of a typical oscillator pulse is shown in fig. 2 . The seed pulses are launched with about $30 \%$ efficiency into the stretcher stage. This is comprised of $450 \mathrm{~m}$ of standard telecommunications type Corning SMF-28 fiber, having a core diameter of $8 \mu \mathrm{m}$, a numerical aperture of 0.13 , and a cutoff wavelength of $1.2 \mu \mathrm{m}$. The second order dispersion $\beta_{2}$ at $1.56 \mu \mathrm{m}$

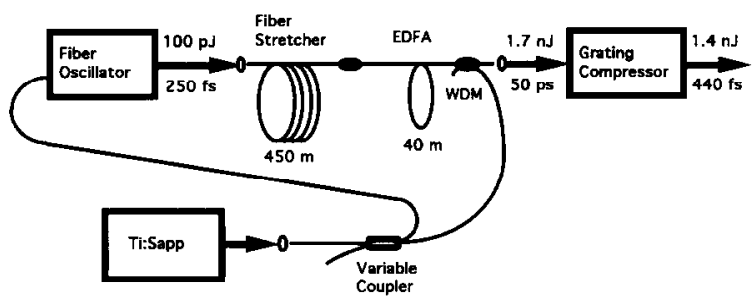

Fig. 1. Schematic of an erbium-doped fiber oscillator/erbiumdoped fiber amplifier system. 

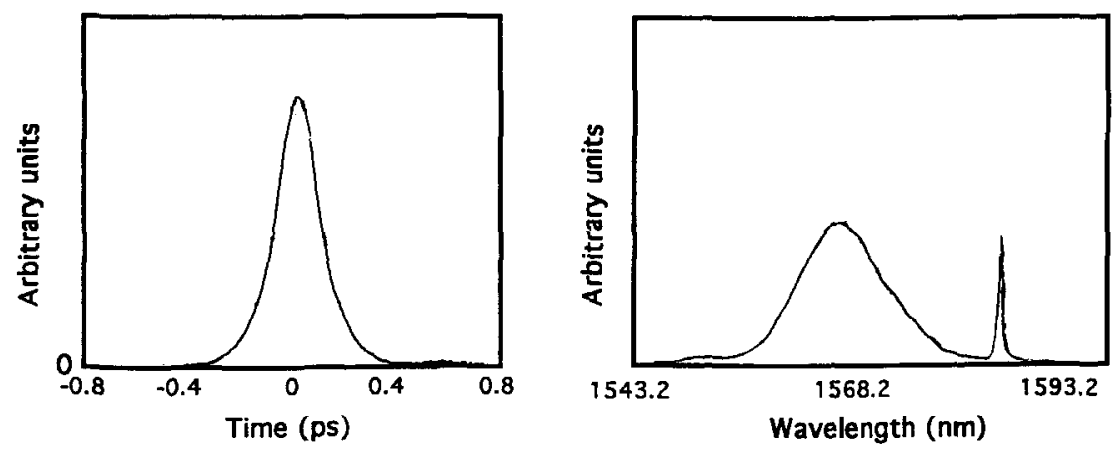

Fig. 2. Autocorrelation trace and spectrum of a seed pulse. The fwhm pulse duration is $250 \mathrm{fs}$ and the fwhm spectral width is $12 \mathrm{~nm}$.

is estimated as $-22000 \mathrm{fs}^{2} / \mathrm{m}$. The resulting stretched pulses are $50 \mathrm{ps}$ in duration. The stretcher fiber is spliced to a low-NA, lightly doped EDFA of $40 \mathrm{~m}$ in length which has similar characteristics to the undoped fiber. The second spectral peak of the EDFA is approximately $12 \mathrm{~nm}$ shorter than the central oscillator wavelength.

Both the oscillator and the amplifier are pumped with $200 \mathrm{~mW}$ of $980 \mathrm{~nm}$ light supplied by a Ti:sapphire laser via a variable coupler, where 60 $\mathrm{mW}$ are directed to the oscillator and the rest is directed to the amplifier. With a pulse energy of $30 \mathrm{pJ}$ launched into the amplifier, ASE is sufficiently suppressed and a single-pass gain of $18 \mathrm{~dB}$ is achieved, resulting in an output energy of $1.7 \mathrm{~nJ}$. Note that the signal does not saturate the amplifier at the pump power level of $140 \mathrm{~mW}$, most likely because the oscillator wavelength is so far from optimum as noted above. We estimate that by increasing the coupled seed signal by about a factor of two almost twice as much power could be extracted from the amplifier.

The amplified pulses are then sent through a double pass, double grating compressor. The gratings have 1200 lines $/ \mathrm{mm}$ groove spacing and are used in near Littrow condition. The compressor configuration uses a set of lenses between the gratings spaced at a distance equal to twice the focal length of one lens. The total grating separation is optimized for maximum compression of the pulses from the amplifier. At this separation, the second order dispersion is calculated to be $20500 \mathrm{fs}^{2} / \mathrm{m}$. The energy efficiency of the grating compressor is approximately $80 \%$, therefore the compressed pulses have an energy content of $1.4 \mathrm{~nJ}$. The shortest compressed pulses obtained are $440 \mathrm{fs}$ in duration as shown in fig. 3 .

A limitation on the ability to get to higher pulse energies in the system described above is governed by the ultimate stretched pulse length from the optical fiber that is able to be compressed with no distortion by the grating compressor. Although secondorder dispersion is compensated in this configuration, third-order dispersion remains uncompensated due to the mismatch between fiber and gratings and leads to a non-uniform chirp. Third-order dispersion is negligible when the pulse-stretching factor is less than $\lambda / \Delta \lambda$, where $\lambda$ is the center wavelength and $\Delta \lambda$ is the bandwidth of the seed pulse. In the case of the oscillator pulses, this value is $\sim 130$. To overcome this limitation, gratings may be used instead of fiber to stretch the pulses.

The next fundamental limit would be that of the generation of self-phase modulation (SPM). For SPM, the nonlinear phase shift is given by

$\phi_{\mathrm{SPM}} \approx n_{2} k_{0} L I_{\mathrm{p}}$

where $n_{2}=3.2 \times 10^{-16} \mathrm{~W} / \mathrm{cm}^{2}, k_{0}=2 \pi / \lambda_{0}, L$ is the length of the fiber and $I_{\mathrm{p}}$ is the peak intensity of the pulse. Inserting typical experimentally obtained values into eq. (1), initially unchirped pulses would experience a nonlinear phase shift of approximately 500 . Within the same $40 \mathrm{~m}$ EDFA, chirped pulses only experience a nonlinear phase shift of 3.5. Generally, the critical nonlinear phase shift is 5 and thus, within this experiment, wc have been able to avoid this critical level, even with a long amplifier fiber. The critical Raman self-scattering threshold should be of about the same order as that of SPM. By stretching input pulses by a factor of 10000 (pos- 

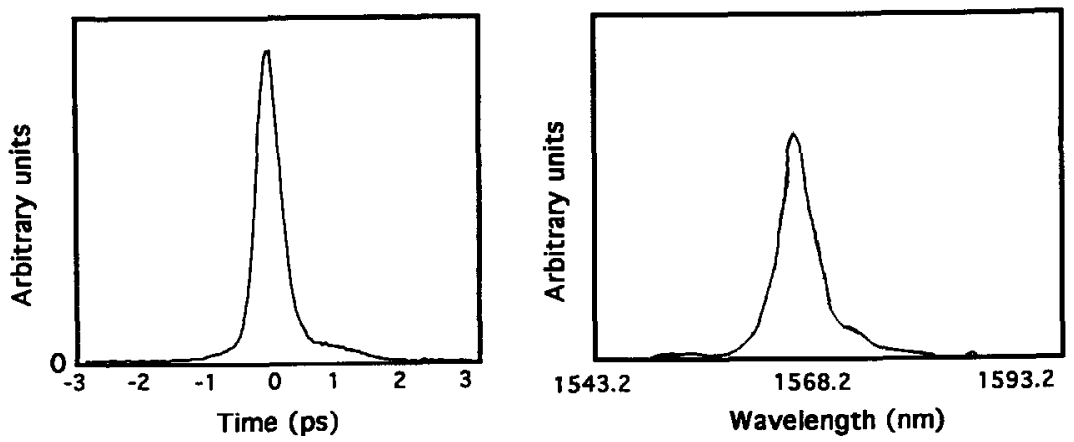

Fig. 3. Autocorrelation trace and spectrum of the shortest recompressed pulse. The fwhm pulse duration is $440 \mathrm{fs}$ and the fwhm spectral width is $6 \mathrm{~nm}$.

sible with a grating stretcher), the nonlinear phase shift generated during amplification in the EDFA described above will not go above the critical level and energies of $200 \mathrm{~nJ}$ can in principle be reached. Higher energy levels would require a shorter amplifier length.

This initial demonstration shows that chirped pulse amplification may be applied to fiber based systems in order to go beyond the pulse energies available directly from fiber oscillators (typically only up to 100 pJ ) and also to avoid the nonlinear processes which occur in fiber amplifiers. Thus, this system can in an optimized design be capable of producing femtosecond pulses of up to the micro-joule level. Such an optimized design would necessarily involve lowering the repetition rate to the order of $1 \mathrm{MHz}$, easily obtainable by lengthening the oscillator. Once the oscillator repetition rate is so lowered, it is possible to use an integrated modulator to create the more optimal repetition rates of approximately a few hundred to a few thousand $\mathrm{Hz}$ to obtain the most efficient energy extraction. A multi-stage amplifier comprised of shorter amplifier fibers and a grating stretcher rather than a fiber stretcher would permit for better compensation of higher-order dispersion terms, allowing for amplification and compression of even shorter pulses than demonstrated here. It should also be pointed out that this system, even as demonstrated, uscs pump light far more efficiently than oscillator designs that are capable of creating higher pulse energies. For such a neodymium-doped fiber oscillator [10], $320 \mathrm{~mW}$ launched pump power is needed to obtain $1 \mathrm{~nJ}$ directly while the above de- scribed hybrid systems can give nearly three times as much pulse energy for the same pump power.

In summary we have demonstrated chirped pulse amplification in fiber power amplifiers for the first time. The technique was employed to greatly improve the pump efficiency of an erbium oscillator power amplifier system. The technique is easily upscalable and with higher pump powers and longer oscillator lengths the generation of femtosecond pulses with energies around $1 \mu \mathrm{J}$ will be possible.

The authors wish to thank Almantas Galvanauskas for technical assistance and Martin Fermann for the use of his oscillator and a critical reading of the manuscript. The authors acknowledge support from the National Science Foundation through the Center for Ultrafast Optical Science under STC PHY 8920108. This work was carried out at IMRA America, Inc. in Ann Arbor, MI.

\section{References}

[1] K.J. Blow, N.J. Doran and D. Wood, J. Opt. Soc. Am. B 5 (1988) 381.

[2] G.P. Agrawal, IEEE Phot. Tech. Lett. 2 (1990) 875.

[3] W. Hodel, D.S. Peter and H.P. Weber, Optics Comm. 97 (1993) 233.

[4] D.J. Richardson, V.V. Afanasjev, A.B. Grudenin and D.N. Payne, Optics Lett. 17 (1992) 1596.

[5] A. Galvanauskas, P. Blixt and J.A. Tellefsen Jr., Appl. Phys. Lett. 63 (1993) 1742.

[6] D. Strickland and G. Mourou, Optics Comm. 56 (1985) 219. 
[8] P.J. Delfyett, L. Florez, N. Stoffel, T. Gmitter, N. Andreadakis, G. Alphonse and W. Ceislik, Optics Lett. 17 (1992) 670 .

[9] M.E. Fermann, L.-M. Yang, M.L. Stock and M.J. Andrejco, Optics Lett. to be published.
[10] G.P. Agrawal, Nonlinear fiber optics (Academic, New York, 1989).

[11] M.H. Ober, M. Hofer and M.E. Fermann, Optics Lett. 18 (1993) 367. 